\title{
Next generation sequencing-based analysis of mitochondrial DNA characteristics in plasma extracellular vesicles of patients with hepatocellular carcinoma
}

\author{
YIJIE LI ${ }^{1 *}, \mathrm{XU} \mathrm{GUO}^{2 *}$, SHANSHAN GUO ${ }^{2}$, YANG WANG $^{1}$, LIN CHEN $^{2}$, YANG LIU $^{2}$, \\ MIN JIA $^{2}$, JIAZE AN $^{1}$, KAISHAN TAO $^{1}$ and JINLIANG XING ${ }^{2}$ \\ ${ }^{1}$ Department of Hepatobiliary Surgery, Xijing Hospital; ${ }^{2}$ State Key Laboratory of Cancer Biology and \\ Department of Physiology and Pathophysiology, Fourth Military Medical University, Xi'an, Shaanxi 710032, P.R. China
}

Received February 5, 2020; Accepted May 14, 2020

DOI: $10.3892 / \mathrm{ol} .2020 .11831$

\begin{abstract}
Emerging evidence has revealed that mitochondrial DNA (mtDNA) is encapsulated in plasma extracellular vesicles (EVs). However, the characteristics of mtDNA in EVs from patients with cancer remain largely unexplored, which greatly limits its clinical application. Whole genome and capture-based sequencing found that EV mtDNA covered the whole mitochondrial genome. The medium fragment size in EV mtDNA was significantly larger compared with that in cell-free mtDNA [cfmtDNA; 159 vs. 109 base pairs (bp); $\mathrm{P}<0.001]$. EV DNA appeared to have a higher mtDNA copy number compared with cfDNA. Of note, patients with hepatitis had >300-bp fragments in EV mtDNA compared with patients with hepatocellular carcinoma (HCC) and healthy controls. EV mtDNA fragments $>300$ bp in length exhibited a significantly higher proportion of EV mtDNA fragment ends than those that were $\leq 300 \mathrm{bp}$ in length in patients with hepatitis. The EV mtDNA copy number in patients with HCC and hepatitis were significantly lower compared with those in healthy controls. Furthermore, inconsistencies in the mtDNA heteroplasmic variant were observed among HCC tissues, plasma and EVs. In conclusion, EV mtDNA exhibited different characteristics among patients with $\mathrm{HCC}$, hepatitis and healthy
\end{abstract}

Correspondence to: Dr Jinliang Xing, State Key Laboratory of Cancer Biology and Department of Physiology and Pathophysiology, Fourth Military Medical University, 169 Changle West Road, Xi'an, Shaanxi 710032, P.R. China

E-mail: xingjl@fmmu.edu.cn

Dr Kaishan Tao, Department of Hepatobiliary Surgery, Xijing Hospital, Fourth Military Medical University, 169 Changle West Road, Xi'an, Shaanxi 710032, P.R. China

E-mail: taokaishan0686@163.com

${ }^{*}$ Contributed equally

Key words: extracellular vesicles, mitochondrial DNA, hepatocellular carcinoma, liquid biopsy controls, indicating the potential value of EV mtDNA as a diagnostic biomarker that complements cfmtDNA.

\section{Introduction}

Extracellular vesicles (EVs) are cell-derived lipid bilayer-enclosed vesicles with a diameter of nanoscale, which can mediate intercellular communication through transferring proteins and nucleic acids derived from donor cells. Almost all types of cells can produce and release EVs, which play complicated roles in various diseases, including cancer (1). Increasing evidence has indicated that EVs secreted by host or tumor cells are extensively involved in tumor development and progression (1-3). An increasing number of studies have been focusing on the diagnostic value of EV detection in various diseases, particularly cancer (4-7).

EVs can carry various cargoes, such as proteins, lipids and nucleic acids (8). Furthermore, previous findings have revealed that EV-derived nuclear DNA (EV nDNA) covers the entire nuclear genome and reflects the mutational status of parental cells $(9,10)$. To date, several studies have detected mutations of EV DNA as a means for early cancer screening, and findings have shown an advantage of EV DNA over cell-free DNA $(11,12)$, as EV DNA it remains relatively intact due to protection of lipid envelop from degradation by DNase (13). Therefore, the characterization of EV DNA in plasma may provide useful biomarkers for the diagnosis and clinical monitoring of cancer.

The mitochondrion is a double membrane-bound organelle found in almost all eukaryotes, which contains its own genetic DNA. Mitochondrial DNA (mtDNA) is a 16,569-base pair (bp) circular chromosome that encodes 13 proteins essential for respiratory energy metabolism. Due to the lack of histone protection, limited DNA repair activities and oxidative stress microenvironment in mitochondria, mtDNA is subject to sequence mutations and copy number changes, which are closely associated with various diseases, especially cancer $(14,15)$. A series of studies have observed a decrease in the mtDNA copy number (termed mtDNA depletion) in several types of cancer, including hepatocellular carcinoma (HCC) (16), which suggests that mtDNA 
depletion may contribute to tumorigenesis. Zhao et al (17) also reported an association between low mtDNA content in peripheral blood leukocytes and high-risk of HBV-associated HCC. Furthermore, our previous study emphasized the critical contributing role of somatic mtDNA D-loop mutations in HBV-associated hepatocarcinogenesis (18). In addition, recent findings have shown that the measurement of plasma cell-free mitochondrial tumor DNA improves the detection of glioblastoma in patient-derived orthotopic xenograft models (19). These findings suggested that mtDNA may reveal unique advantages in tumor diagnosis, treatment monitoring and evaluation of prognosis.

The full mitochondrial genome was observed in EVs (20). Furthermore, Sansone et al (21) have reported that EVs can package and transfer mtDNA into metabolically damaged breast cancer cells, thereby restoring their metabolic activity and leading to endocrine treatment resistance. Findings of the aforementioned studies suggested that, compared with EV nDNA, EV mtDNA may be a novel cancer detection marker. However, to date, the full characteristics of mtDNA in EVs remain largely unexplored, which greatly limits the clinical application of EV mtDNA detection in patients with cancer.

In the present study, next-generation sequencing was used to profile the entire EV DNA from patients with HCC. Moreover, to the best of our knowledge, the EV mtDNA characteristics in patients with $\mathrm{HCC}$, hepatitis and healthy controls were systematically analyzed and compared for the first time, laying a foundation for the potential clinical application of EV mtDNA as a liquid biopsy biomarker.

\section{Materials and methods}

Sample collection. A total of 15 patients with HBV-associated $\mathrm{HCC}$, five patients with hepatitis with HBV infection and five healthy controls were recruited from Xijing Hospital, Fourth Military Medical University (FMMU) in Xi'an, China between April 2018 and September 2019. Patients with pathologically diagnosed HCC with HBV were recruited, and there were no other comorbidities, such as HCV or HIV infection. For patients with hepatitis with HBV infection, no cirrhosis was observed by B-ultrasound.

Peripheral venous blood (10 $\mathrm{ml}$ per subject) was collected from patients with hepatitis, healthy controls and patients with HCC who had not received any treatment (such as radiofrequency ablation, hepatectomy or transcatheter arterial chemoembolization) prior to blood sample collection. Paired tumor tissues and adjacent non-HCC tissues were collected in five patients HCC who had undergone hepatectomy. The study was approved by the Ethics Committee of FMMU and written consent was obtained from each subject.

The clinical data of all subjects was obtained from medical records for analysis, including: Personal data (age at diagnosis and sex), blood test results (alphafetoprotein, aspartate aminotransferase, alanine aminotransferase, $\gamma$-glutamyl transpeptidase, total bilirubin, alkaline phosphatase, albumin), Tumor-Node-Metastasis (TNM) stage and cirrhosis status. TNM stage referred to TNM Staging System of AJCC (8th version) (22). Patient characteristics are listed in Table SI.
Isolation of EVs from plasma samples. Peripheral blood was drawn from the median cubital vein in the antecubital fossa into EDTA-containing tubes and centrifuged at $300 \mathrm{x} \mathrm{g}$ for 15 min to collect plasma within $2 \mathrm{~h}$. Plasma samples were centrifuged again at $11,200 \mathrm{x}$ g for $30 \mathrm{~min}$ to remove apoptotic bodies, mitochondrial particles and large cell debris. Next, $\sim 4 \mathrm{ml}$ of plasma was centrifuged at $110,000 \mathrm{x} \mathrm{g}$ for $8 \mathrm{~h}$. All centrifugation was performed at $4^{\circ} \mathrm{C}$. The resulting $\mathrm{EV}$ pellet was suspended in $50 \mu \mathrm{l} \mathrm{PBS}$ and stored at $-20^{\circ} \mathrm{C}$ for further use. The identification of EV was performed by transmission electron microscopy, nanoparticle tracking analysis and western blot analysis (Fig. S1).

Transmission electron microscopy. Similar to the previous description (23), freshly isolated plasma EVs were dissolved in PBS buffer, dropped into a carbon-coated copper grid, where they dried at room temperature. Then, the EVs were subjected to negative staining with $1 \%$ uranyl acetate at room temperature for $1 \mathrm{~min}$ and washed twice with deionized water. After dried at room temperature, the EVs were imaged by JEM-1400Plus transmission electron microscope (JEOL, Inc).

Nanoparticle tracking analysis. Nanoparticle tracking analysis (NTA) was to detect the size distribution and concentration of the plasma EVs using ZetaView version 8.04.02 (Particle Metrix, Inc). Isolated plasma EVs were diluted with PBS buffer to 1:100 and resuspended before being injected into the sample cell chamber.

Western blotting. Plasma EV isolations were lysed with RIPA buffer (Beijing Solarbio Science \& Technology Co., Ltd.). The protein concentration in the EV lysate solution was determined using the BCA protein assay kit (Beyotime Biotechnology, Inc). Proteins $(30 \mu \mathrm{g})$ from plasma EVs were loaded per lane onto a $10 \%$ gel, resolved using SDS-PAGE and then transferred to PVDF membranes (Bio-Rad, Inc). The PVDF membranes were blocked with $5 \%$ non-fat milk in PBS for $1 \mathrm{~h}$ at room temperature, and then probed separately with anti-human CD9 (1:2,000, cat. no. ab92726, Abcam), anti-human CD63 (1:1,000, cat. no. ab134045, Abcam) and anti-human CD81 (1:1,000, cat. no. ab109201, Abcam) for $16 \mathrm{~h}$ at $4^{\circ} \mathrm{C}$. After washing, the blots were incubated with horseradish peroxidase-conjugated goat anti-rabbit secondary antibodies (1:10,000, cat. no. SA00001-2, ProteinTech Group, Inc) for $1 \mathrm{~h}$ at room temperature. Protein signals were visualized using Chemiscope 3000 mini chemiluminescence imaging system and Chemi Capture software (version 16.12.03A. Clinx, Inc. URL: http://www.clinx.cn/case_cat/info?id=49).

DNA extraction from EV, plasma and tissue samples. To eliminate the contamination of ss- and ds-DNA, each EV pellet sample was treated with 2U DNaseI (Thermo Fisher Scientific, Inc.) for $30 \mathrm{~min}$ at $37^{\circ} \mathrm{C}$ prior to DNA extraction, and then DNaseI was heat-inactivated by incubation for $10 \mathrm{~min}$ at $70^{\circ} \mathrm{C}$. Subsequently, DNA was extracted from EV pellets using the QIAamp DNA micro kit (Qiagen $\mathrm{AB}$ ) and cfDNA was extracted from an average of $2 \mathrm{ml}$ plasma using the QIAamp Circulating Nucleic Acid kit (Qiagen AB), following the manufacturer's instructions. In addition, DNA was extracted from tissue samples using the E.N.Z.A DNA kit (Omega Bio-Tek, Inc.) following 
the manufacturer's instructions. DNA quantity and quality were analyzed using Qubit 3.0 (Thermo Fisher Scientific, Inc.) and Agilent 2100 bioanalyzer system (Agilent Technologies $\mathrm{GmbH}$ ).

Library construction and whole genome sequencing (WGS). NEB ultra v2 kit (New England Biolabs, Inc.) was used for the construction of a sequencing library of tissue DNA, cfDNA and EV DNA, following the manufacturer's instructions. WGS was carried out for cfDNA and EV DNA sequencing libraries of five HCC, five hepatitis and five healthy control samples on the Hiseq X Ten platform (Illumina, Inc.) with a paired-end read length of $150 \mathrm{bp}$.

Capture-based mtDNA sequencing. Sequencing libraries of matched cfDNA, EV DNA and tissue DNA from 5 HCC patients were mixed with home-made biotinylated capture probes at a ratio of 1:800 for hybridization, as previously described (18), with minor modifications. Probes with an average length of $250 \mathrm{bp}$ specifically targeted to mtDNA and 3 nuclear genes were captured. Finally, the captured libraries which contained cfmtDNA, EV mtDNA and tissue mtDNA were sequenced on the Hiseq X Ten platform (Illumina, Inc.) using paired-end runs of $2 \times 150 \mathrm{bp}$.

mtDNA copy number analysis. Based on WGS data, the absolute mtDNA copy number was calculated as the average sequencing depth of mtDNA divided by the average sequencing depth of total DNA x2.

Bioinformatics analysis of sequencing data. Raw data were trimmed using Fastp (version 0.20.0) (24) to remove adapter sequences and reads whose lengths were $<50 \mathrm{bp}$ and mean quality $<$ Q30. BWA (version 0.7.10-r789) was used to map clean data (25). To minimize the effect of nuclear mtDNA segments, clean data were initially mapped to the Revised Cambridge Reference Sequence of mtDNA and whole genome hg19. Next, Picard tools (version 2.18.27 https://github.com/broadinstitute/picard/releases/tag/2.18.27) were used to mark and remove the duplicate reads. Local realignment was performed using IndelRealigner in GATK (version 3.2-2) (26). Finally, the pileup files were generated by Samtools (version 1.8) (25) for heteroplasmic variants screening under the following filter conditions: i) A minimum allele frequency of $\geq 1 \%$ on both strands; ii) $\geq 3$ reads in each strand supporting the alternative allele; iii) a total sequencing coverage of $\geq 100 \mathrm{X}$.

Statistical analysis. The data are presented as the mean \pm standard error of the mean. GraphPad Prism 7.0 (GraphPad Software, Inc.) and SPSS 21.0 (IBM, Inc.) were used for statistical analysis. Statistical differences between two groups were analyzed by unpaired t-test. Differences between multiple groups were analyzed using one-way ANOVA with post hoc test by least-significant difference. All P-values were two-tailed. $\mathrm{P}<0.05$ was considered to indicate a statistically significant difference.

\section{Results}

EV mtDNA has a larger fragment size compared with cell-free mtDNA (cfmtDNA). Based on WGS data, the distribution of DNA fragment size was analyzed. The results showed that the cfmtDNA and EV mtDNA had a dominant fragment size peak at $\sim 80$ and $\sim 130 \mathrm{bp}$, respectively (Fig. 1A). The EV mtDNA exhibited a larger median fragment size than cfmtDNA (159 bp vs. 109 bp; $\mathrm{P}<0.001$; Fig. 1B). Furthermore, the data indicated that EV mtDNA exhibited a significantly smaller proportion of short fragments $(0-100 \mathrm{bp})$ compared with cfmtDNA $(\mathrm{P}<0.001$; Fig. 1C). By contrast, more long fragments (201-300 and $>300 \mathrm{bp}$ ) were significantly enriched in EV mtDNA compared to cfmtDNA $(\mathrm{P}<0.001$ and $\mathrm{P}=0.001$, respectively; Fig. 1C). Similar to mtDNA, EV nDNA also exhibited a significantly larger fragment size compared with cfDNA $(\mathrm{P}<0.001$; Fig. 1D-E). In addition, an obvious peak in fragment size at $\sim 330$ bp was observed in EV nDNA, but not cfDNA, which might be due to double-nucleosome DNA fragmentation (Fig. 1D). As expected, more long fragments (201-300 and $>300 \mathrm{bp}$ ) were significantly enriched in EV nDNA than in cfDNA $(\mathrm{P}=0.049$ and $\mathrm{P}=0.001$; Fig. $1 \mathrm{~F})$.

EV mtDNA fragment size in patients with hepatitis is larger compared with that in patients with HCC and healthy controls. The distribution of EV DNA fragment size was further compared in plasma samples from healthy controls, patients with hepatitis and patients with HCC. The data indicated that EV mtDNA from patients with hepatitis exhibited a significantly larger fragment size compared with that from patients HCC and healthy controls (Fig. 2A and C). The medium fragment size of EV mtDNA was 163, 145 and 168 bp in HCC patients, healthy controls and hepatitis patients, respectively (Fig. 2B). Furthermore, the interval distribution of the fragment size was analyzed (Fig. 2C), and it was found that the proportion of EV mtDNA fragments with a length of 101-200 bp in patients with hepatitis was significantly lower compared with that in HCC patients $(\mathrm{P}=0.049)$ and healthy controls $(\mathrm{P}=0.019)$. Conversely, the proportion of EV mtDNA fragments with a length of $>300 \mathrm{bp}$ in patients with hepatitis was significantly higher compared with that in patients HCC $(\mathrm{P}=0.003)$ and healthy controls $(\mathrm{P}<0.001)$. However, no significant difference in fragment size distribution in EV nDNA was observed among patients HCC, hepatitis and healthy controls (Fig. 2D-F).

EVmtDNA covers the whole mitochondrial genome. To confirm whether the whole mitochondrial genome can be encapsulated in EVs from patients HCC and healthy controls, the sequencing reads of EV mtDNA were mapped to the human reference mitochondrial genome, and the coverage depth of each EV mtDNA site was then divided by the average sequencing depth of the whole mtDNA to eliminate the deviation caused by the different sequencing depths among samples. Similar to cfmtDNA (Fig. 3A), EV mtDNA covered the whole mitochondrial genome (Fig. 3B) with no obvious preference in DNA regions, suggesting a non-specific encapsulation; however, the mechanism underlying mtDNA encapsulation remains largely unknown. Furthermore, no significant difference in mtDNA encapsulation was observed among patients HCC, healthy controls and patients with hepatitis (Fig. 3C-E).

Distribution of mtDNA ends in EV DNA. The distribution of mtDNA fragment cleavage position was explored and mtDNA ends were defined as the cleavage position of mtDNA 

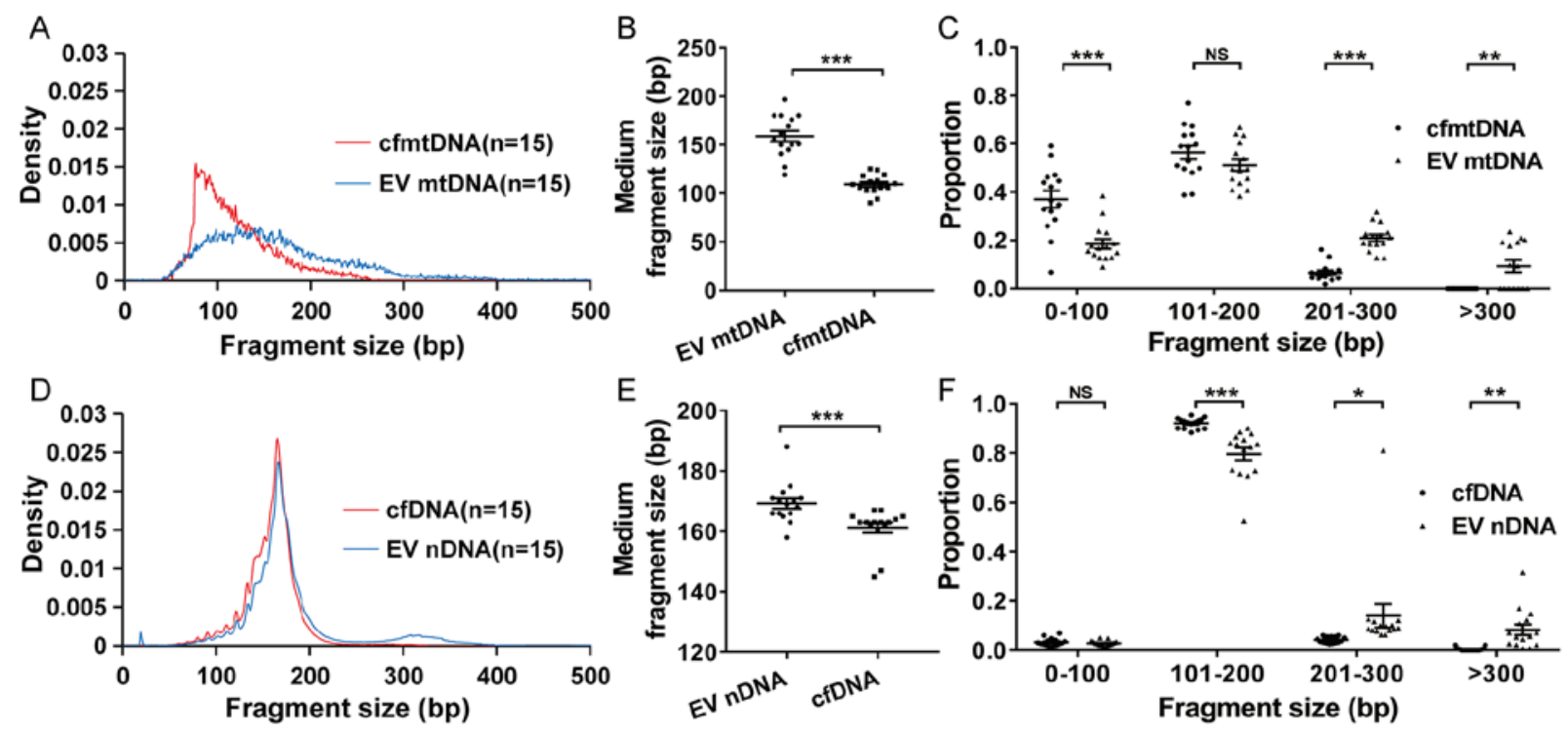

Figure 1. Distribution of DNA fragment size in plasma and EV samples. (A) Density, (B) medium fragment size and (C) proportion of cfmtDNA and EV mtDNA fragments with different sizes. Density was defined as the fragment number of a certain length divided by the total fragment number. Proportion was defined as the fragment number of a certain length range divided by the total fragment number. (D) Density, (E) medium fragment size and (F) proportion of cfDNA and EV nDNA fragments with different sizes. EV nDNA, extracellular vesicle nuclear DNA. ${ }^{*} \mathrm{P}<0.05,{ }^{* *} \mathrm{P}<0.01$ and ${ }^{* * * *} \mathrm{P}<0.001$. NS, no significance; EV, extracellular vesicles; mtDNA, mitochondrial DNA; cfmtDNA, cell-free mtDNA; EV mtDNA, extracellular vesicle mtDNA; bp, base pair; nDNA, nuclear DNA.
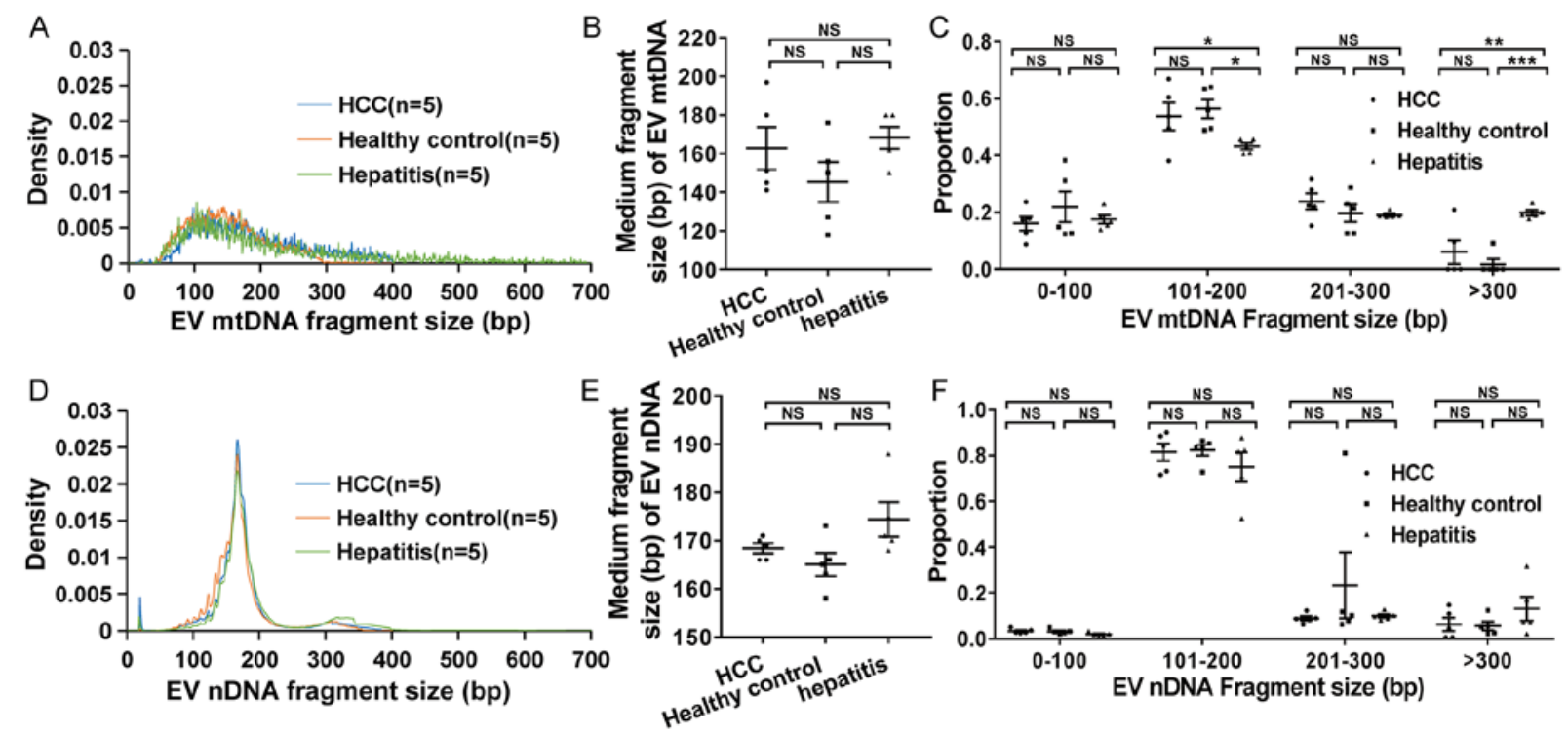

Figure 2. Comparison of DNA fragment size among patients with HCC, hepatitis and healthy controls. (A) Density, (B) medium fragment size and (C) proportion of EV mtDNA fragments with different sizes. (D) Density, (E) medium fragment size and (F) proportion of EV nDNA fragments with different sizes. ${ }^{*} \mathrm{P}<0.05,{ }^{* *} \mathrm{P}<0.01$ and ${ }^{* * * *} \mathrm{P}<0.001$. NS, no significance; HCC, hepatocellular carcinoma; mtDNA, mitochondrial DNA; EV mtDNA, extracellular vesicle mtDNA; nDNA, nuclear DNA.

fragments at the mitochondrial genome. When compared with the majority of mtDNA sites, a small part of mtDNA sites showed a higher proportion of ends (range, 0.0004-0.0010) in EV mtDNA (Fig. 4A-D). However, these mtDNA sites did not exist at the same time in different samples. The number of mtDNA end sites that were cleaved more than twice and the cleavage number of these sites in EV mtDNA from different sample groups were further compared. It was found that healthy controls had a significantly higher site and cleavage number of mtDNA ends compared with patients with $\mathrm{HCC}(\mathrm{P}=0.005$ and $\mathrm{P}=0.003)$ and hepatitis $(\mathrm{P}=0.021$ and $\mathrm{P}=0.020$; Fig. 4E).
EV mtDNA fragments of $>300$ bp exhibited a significantly higher proportion of EV mtDNA ends compared with those with $\leq 300$ bp in patients with hepatitis $(\mathrm{P}<0.001$; Fig. $4 \mathrm{~F})$.

mtDNA copy number analysis of plasma EV DNA. The mtDNA copy number was compared among different types of DNA samples, and it was found that HCC tissues had a significantly higher mtDNA copy number (mean, 681.17) compared with cfDNA (mean, 2.89; $\mathrm{P}<0.001$ ) and EV DNA (mean, 9.14; P<0.001; Fig. 5A). It appeared that EV DNA had a significantly higher mtDNA copy number than cfDNA 

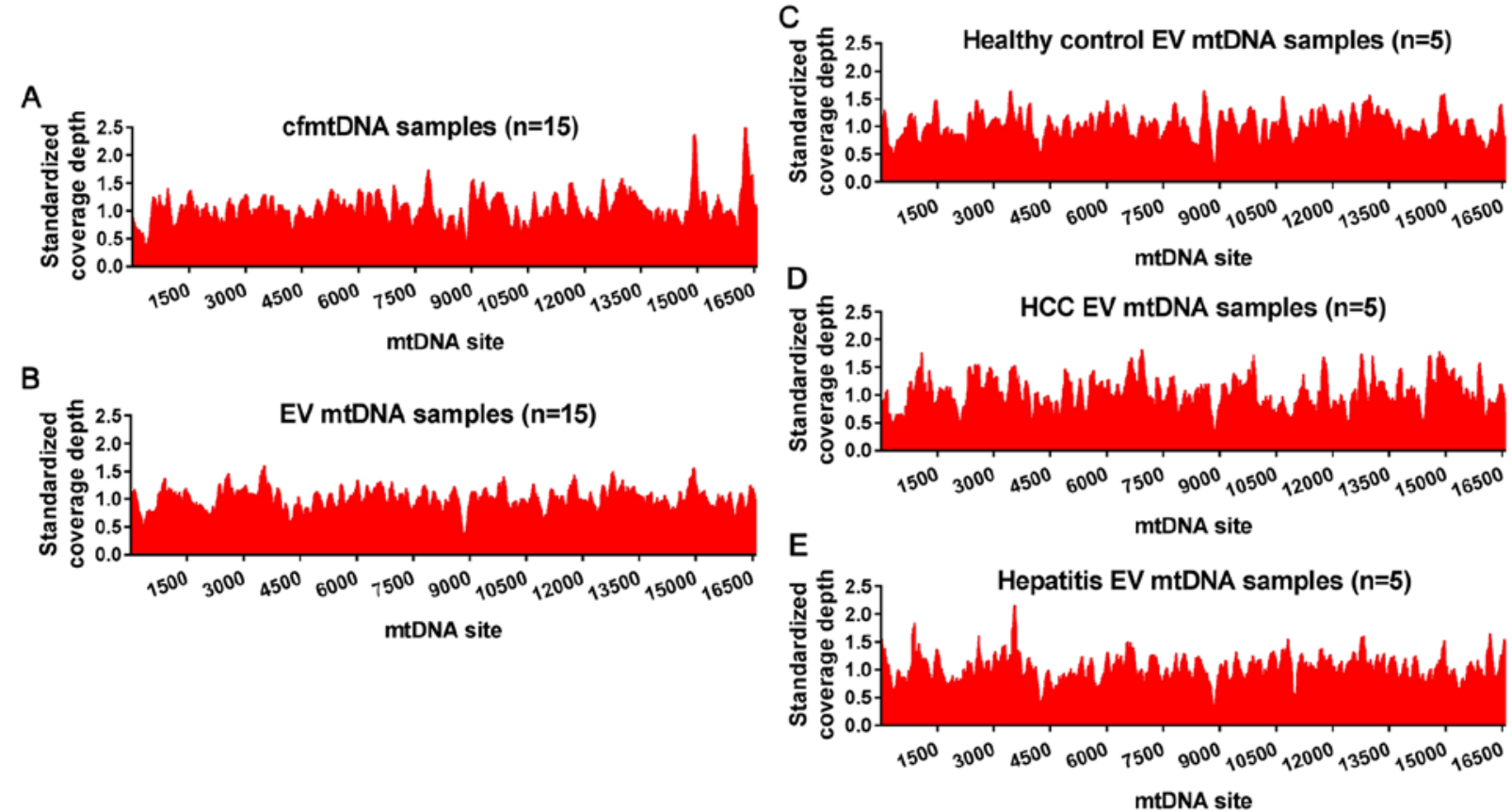

Figure 3. Coverage analysis of EV mtDNA and cfmtDNA. (A) Standardized coverage depth of cfmtDNA in 15 samples. Standardized coverage depth of EV mtDNA in (B) all subjects, (C) healthy controls, patients with (D) HCC and (E) hepatitis. Coverage depth was standardized by the average sequencing depth of whole mtDNA. mtDNA, mitochondrial DNA; EV mtDNA, extracellular vesicle mtDNA; cfmtDNA, cell-free mtDNA; HCC, hepatocellular carcinoma.
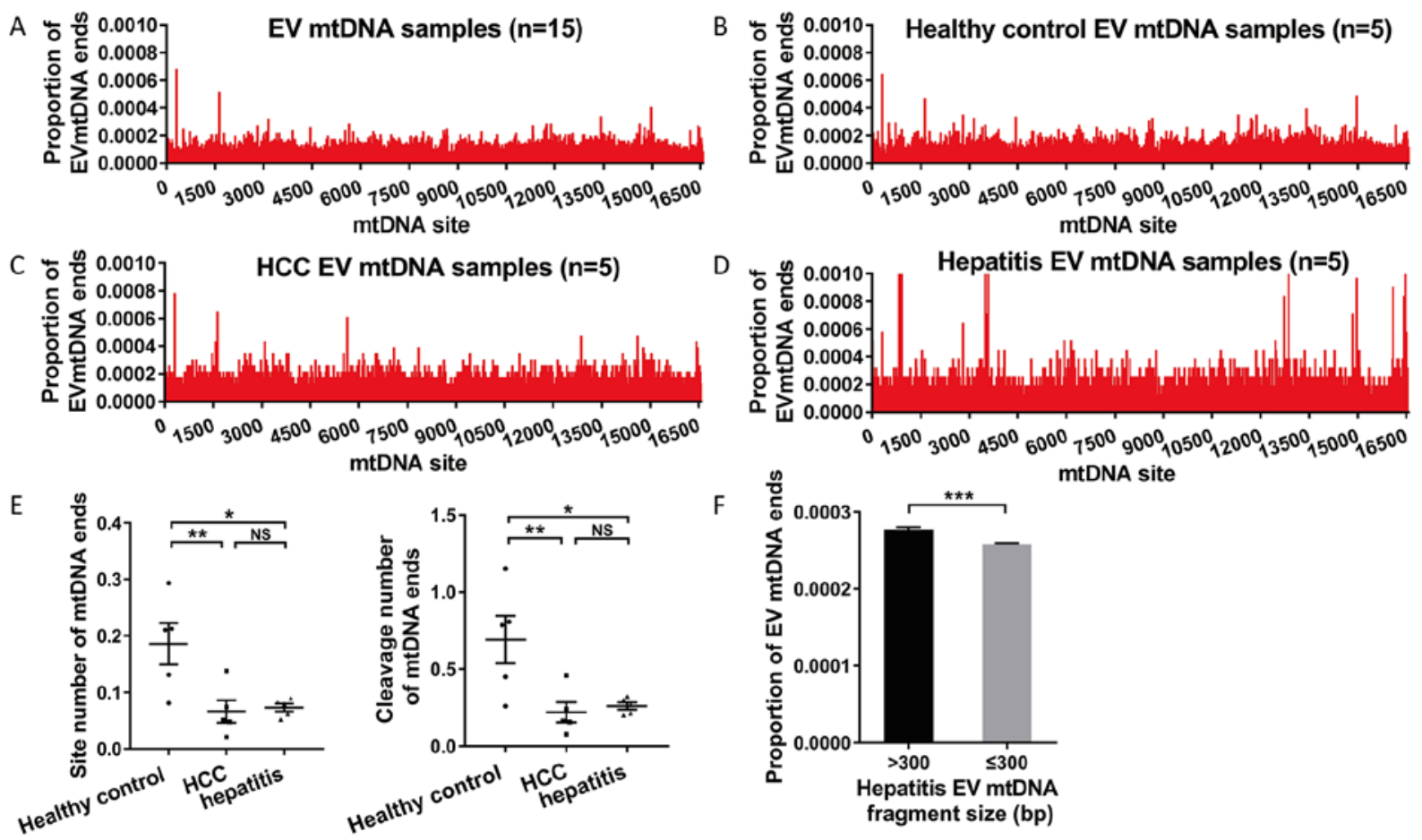

Figure 4. Distribution of mtDNA ends in EV DNA. (A) Proportion of EV mtDNA ends in 15 samples. Proportion of EV mtDNA ends in (B) healthy controls, patients with (C) HCC and (D) hepatitis. (E) Site number of mtDNA ends and cleavage number of mtDNA ends in healthy controls, patients HCC and hepatitis. mtDNA ends were defined as the cleavage position of mtDNA fragments at the mitochondrial genome. The site number of mtDNA ends was defined as the total number of sites that were cleaved more than twice. The cleavage number of mtDNA ends was defined as the number of ends in the site that was cleaved more than twice. (F) EV mtDNA ends proportion of fragments $>300$ and $\leq 300$ bp in length in patients with hepatitis. ${ }^{*} \mathrm{P}<0.05$, ${ }^{* * *} \mathrm{P}<0.01$ and ${ }^{* * * *} \mathrm{P}<0.001$. NS, no significance; mtDNA, mitochondrial DNA; EV mtDNA, extracellular vesicle mtDNA; HCC, hepatocellular carcinoma.

$(\mathrm{P}=0.017)$. Furthermore, healthy controls exhibited a significantly higher mtDNA copy number (mean, 20.63) than both hepatitis (mean, 5.41; $\mathrm{P}=0.002$ ) and HCC patients (mean, 9.14;
$\mathrm{P}=0.014)$. However, no significant difference in the mtDNA copy number was observed between patients with $\mathrm{HCC}$ and hepatitis ( $\mathrm{P}=0.367$; Fig. 5B). 

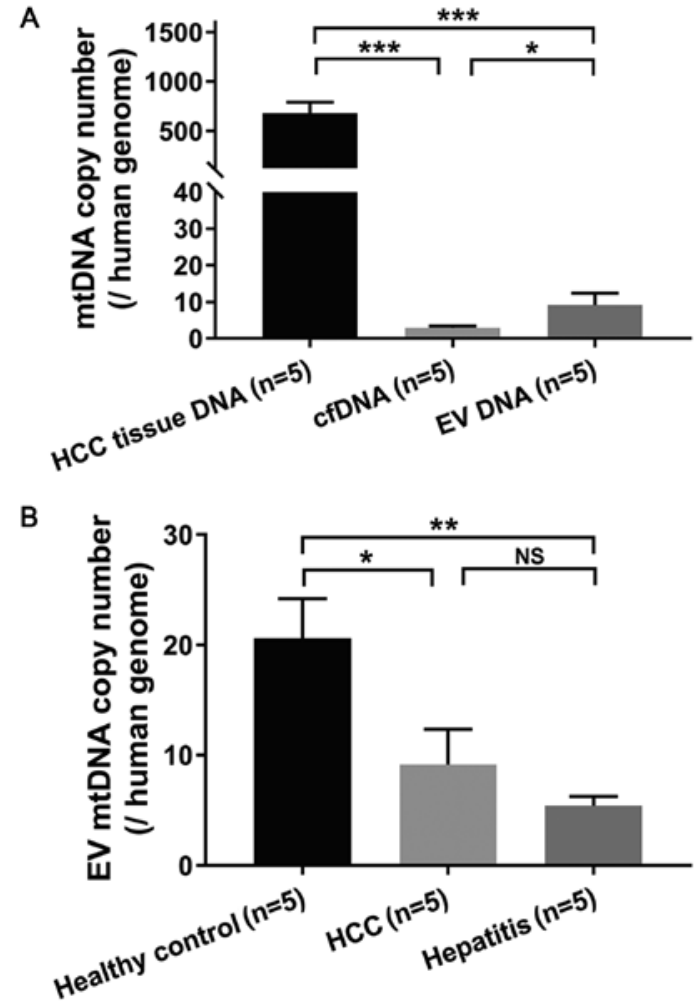

Figure 5. mtDNA copy number analysis of plasma EV DNA. (A) Comparison of mtDNA copy number among tumor tissue, plasma and EVs from patients with HCC. (B) Comparison of EV mtDNA copy number among healthy controls, patients with HCC and hepatitis. ${ }^{*} \mathrm{P}<0.05,{ }^{* *} \mathrm{P}<0.01$ and ${ }^{* * * *} \mathrm{P}<0.001$ NS, no significance; mtDNA, mitochondrial DNA; EV DNA, extracellular vesicle DNA; HCC, hepatocellular carcinoma.

Consistent analysis of mtDNA heteroplasmic variants in the tissue, plasma and EVs of the same patient. The consistency of mtDNA heteroplasmic variants was investigated in different samples from five patients with HCC (Fig. 6). A total of 252 mtDNA heteroplasmic variants were detected in all the samples. The majority of mtDNA heteroplasmic variants were identified in plasma cfmtDNA (131 variants) and EV mtDNA (150 variants). Among them, 77 heteroplasmic variants existed in both EV mtDNA and cfmtDNA. A total of $25 \mathrm{mtDNA}$ heteroplasmic variants were found in tumor tissues, seven of which were detected in the plasma and 8 in EVs. Only three out of 37 heteroplasmic variants that derived from non-HCC tissues could be detected in plasma and EVs.

\section{Discussion}

In the present study, it was found that EV mtDNA covered the whole mitochondrial genome. The characteristics of EV mtDNA were significantly different from those of cfmtDNA. Disease status in the liver (HBV-associated hepatitis and HCC) markedly affected the EV mtDNA characteristics. In addition, mtDNA mutation detection of EV DNA was complementary to cfDNA and tissue DNA samples. To the best of our knowledge, the present study is the first study to systematically analyze the characteristics of EV mtDNA in patients with HCC.

The present data showed that EV mtDNA had a larger fragment size compared with cfmtDNA, and a consistent result was also observed in EV nDNA. Similar to the result of the present study, two previous studies have reported a larger fragment size in EV nDNA compared with in cfDNA, and even fragments with a size range of 2.5-10 kbp have been found to exist in EV nDNA $(9,10)$. These findings are likely to be associated with the unique vesicle structure of EVs, which protects internal DNA from external interference (27). In addition, the different fragment size distribution between mtDNA and nuclear DNA in EVs and plasma suggests the existence of different degradation mechanisms. cfDNA fragments commonly show a prominent mode average at $\sim 160$ bp, suggesting release from apoptotic caspase-dependent cleavage (28). However, the underlying mechanism of mtDNA degradation needs to be further explored.

Evidence has shown that cfDNA analysis is a promising tool with potential for broad application in clinical settings, especially cancer detection. Recent findings have shown that the cell of origin and the mechanism of cfDNA release into the blood can mark cfDNA with specific fragmentation signatures, demonstrating that differences in fragment lengths of cfDNA may be exploited to enhance sensitivity in detecting the presence of circulating tumor DNA $(29,30)$. However, whether fragment size analysis of mtDNA in cfDNA and EV DNA contributes to cancer detection is largely unknown. Previous studies have found that the release of mtDNA from mitochondria depends on the mitochondrial permeability transition pore (MPTP), BAK and BAX proteins (31-33). MPTP is located in the mitochondrial inner membrane. Inhibition of MPTP opening by cyclosporine A decreases leakage of mtDNA into cytosol (31). In hepatitis caused by HBV infection, HBV induces MPTP opening to regulate the release of mitochondrial contents, including mtDNA (32). When BAK and BAX are activated, they oligomerize in the mitochondrial outer membrane, which increases the permeability of mitochondrial membrane and leads to the release of mtDNA and cytochrome c (33). In the present study, it was found that the long mtDNA fragment more frequently existed in EV DNA from patients with hepatitis compared with HCC and healthy controls. This suggested that hepatitis may affect the permeability of the mitochondrial membrane, resulting in the release of longer mtDNA fragments into the cytosol and reducing the degradation of mtDNA in EVs. In addition, the analysis of mtDNA ends in EVs also indicated that patients with hepatitis and HCC exhibited a lower site and cleavage number of mtDNA ends compared with healthy controls, suggesting a more uneven degradation. Furthermore, it was found that long EV mtDNA fragments $(>300 \mathrm{bp})$ exhibited a significantly higher proportion of EV mtDNA ends in patients with hepatitis, suggesting that a non-random end model may exist in inflammatory cells. These findings indicated that the fragment size and end analysis of EV mtDNA may be used as a biomarker for liver inflammation. Based on the present findings, it was speculated that EV mtDNA may also have fragment length and mtDNA terminal end characteristics in other tumors. However, because of hepatitis stage during the development of HBV-associated HCC, EV mtDNA characteristics in other tumors may be different from those in HCC $(34,35)$. In addition, whether mtDNA in EVs have a critical role in inflammatory diseases of the liver needs to be further explored. 
Patient 1

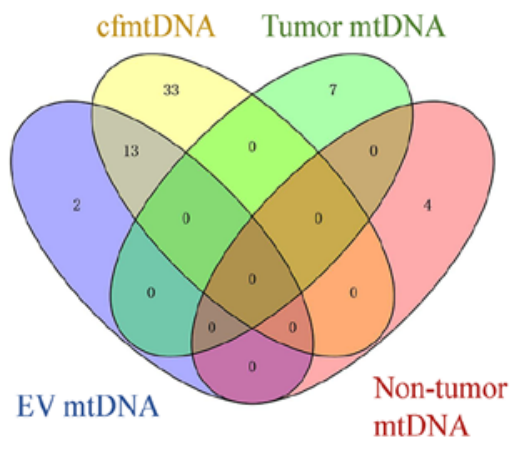

Patient 4

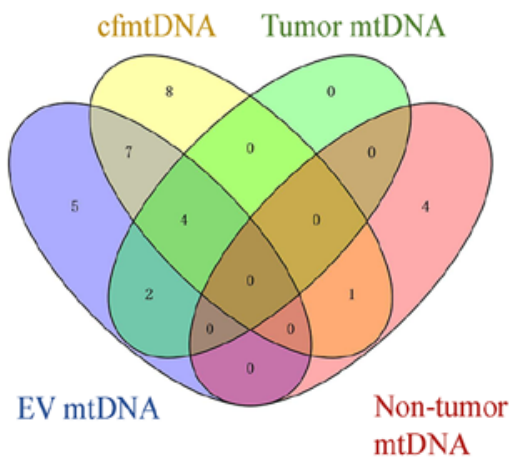

Patient 2

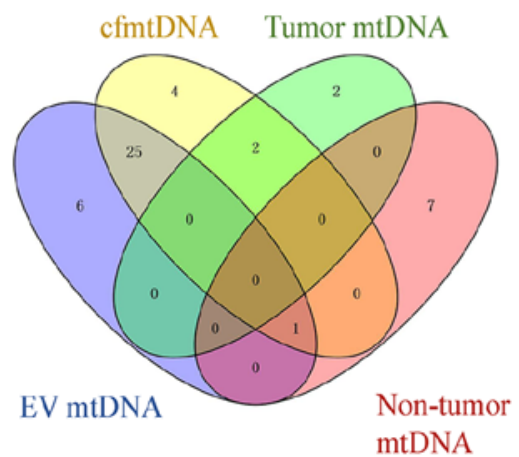

Patient 5

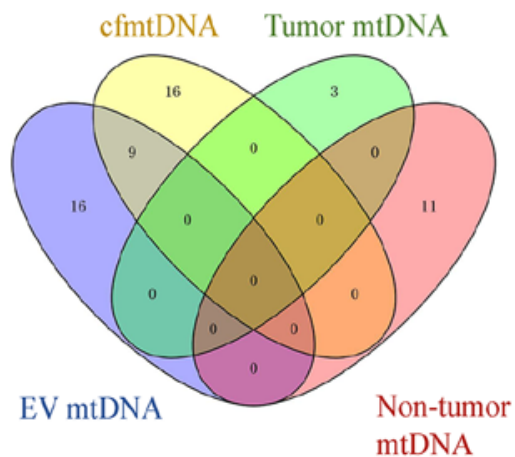

Patient 3

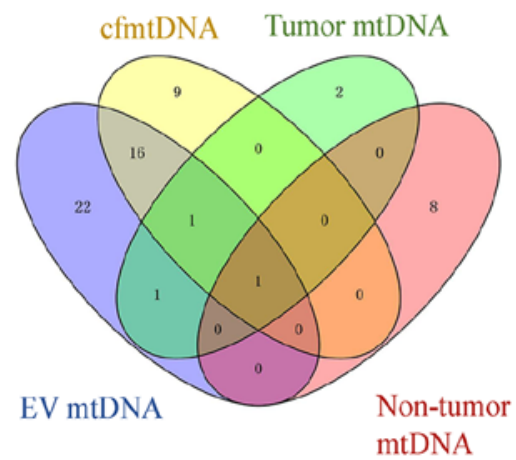

Figure 6. Consistent analysis of mtDNA heteroplasmic variants from tumor tissue, non-tumor tissue, EV mtDNA and cfmtDNA. mtDNA, mitochondrial DNA; EV mtDNA, extracellular vesicle mtDNA; cfmtDNA, cell-free mtDNA.

Compared with nuclear DNA, mtDNA has higher copy numbers in each cell, a shorter genome length and an active transcription and translation. Changes in the mtDNA copy number have been extensively explored in a variety of tumors, including HCC. Previous findings have shown a decrease in mtDNA copy number in HCC tissues, which may contribute to HCC tumorigenesis and progression (36). Similar results were observed in the present study, where the mtDNA copy number was significantly decreased in EV DNA from patients with HCC and hepatitis compared with that from healthy controls. In addition, both EV DNA and cfDNA exhibited a significantly lower mtDNA copy number, when compared with HCC tissue DNA, suggesting a faster degradation of mtDNA over nuclear DNA in both EVs and plasma. Consistent with the protective role of EVs, EV DNA had a slightly higher copy number compared with cfDNA. A recent study also showed a higher concentration of mtDNA in EVs compared with plasma (37). The present study also confirmed that EV DNA covered the whole mitochondrial genome, suggesting that EV mtDNA can carry all the information from tumor cells and may be used as a potential cancer biomarker. Overall, these findings suggest that detection of the EV mtDNA copy number should be considered in the clinical diagnosis of HCC. An increased sample size is required to validate the present results in the future.

Recently, an increasing number of studies have focused on the detection of EVs and its potential clinical application value. Bernard et al (38) reported that KRAS mutations in exosome DNA are more consistent with KRAS mutations in tissue DNA compared with cfDNA in primary pancreatic cancer. Two more studies have also indicated that EV-derived DNA is superior to
cfDNA for specific mutation detection in pancreatic (12) and non-small-cell lung cancer (11). At present, there is no study investigating the profile of mtDNA mutations in EVs. The present study systematically described the mtDNA mutations in different type of samples from patients with HCC (Fig. 6). The data showed that more mtDNA heteroplasmic mutations were detected in EVs and plasma compared with in paired tissues. However, compared with the HCC and adjacent non-HCC tissue, the majority of mtDNA heteroplasmic variants were found specifically in the plasma and EVs, which may come from white blood cells or other tissues. However, this remains to be confirmed. A recent study reported that tumor-derived mtDNA mutations are rarely detected in cfDNA (39). The present results also indicated that only a small number of tumor-derived mtDNA heteroplasmic variants can be detected in cfDNA and EV DNA. These findings suggested that EV mtDNA mutation detection may provide an alternative choice for cancer diagnosis and clinical monitoring. These data also showed that EV DNA was a good complement to plasma cfDNA in the heteroplasmic variant detection of mtDNA.

In conclusion, EV mtDNA exhibited different characteristics among patients with HCC and hepatitis and healthy controls, indicating its potential clinical application value as a diagnostic biomarker that complements cfmtDNA. However, the present study also had limitations. For example, the sample size was small, therefore the present results need to be validated using a larger sample size to further demonstrate the application of EV mtDNA characteristics in the diagnosis of HCC. Meanwhile, due to the large amount of data and high cost of the whole genome sequencing, capture-based mtDNA sequencing could be used to detect EV mtDNA characteristics, and the value of this technique needs to be verified. 
Further functional studies are also needed to understand the underlying mechanisms suggested by the present findings.

\section{Acknowledgements}

Not applicable.

\section{Funding}

This work was supported by the National Natural Science Foundation of China (grant nos. 81602463 and 8183000102) and the Science and Technology Co-ordinate Innovation Project of Shaanxi Province, China (grant nos. 2016KTZDSF-01-02, 2017SF-188 and 2018ZDXM-SF-061).

\section{Availability of data and materials}

The datasets used and/or analyzed during the present study are available from the corresponding author on reasonable request.

\section{Authors' contributions}

JX and KT conceived and designed the study. YJL, YW and YL performed the experiments. JA, LC and MJ collected clinical samples and experimental data. YJL, XG and SG analyzed the data and interpreted the data. YJL, LC, MJ and JA wrote the manuscript. All authors read and approved the final manuscript.

\section{Ethics approval and consent to participate}

The study was approved by the Ethics Committee of the Fourth Military Medical University (Xi'an, China; approval no. KY20163277-1). Written consent was provided by each patient.

\section{Patient consent for publication}

Not applicable.

\section{Competing interests}

The authors declare that they have no competing interests.

\section{References}

1. Tkach $\mathrm{M}$ and Thery C: Communication by extracellular vesicles: Where we are and where we need to go. Cell 164: 1226-1232, 2016.

2. Li X, Li C, Zhang L, Wu M, Cao K, Jiang F, Chen D, Li N and $\mathrm{Li} \mathrm{W}$ : The significance of exosomes in the development and treatment of hepatocellular carcinoma. Mol Cancer 19: 1, 2020.

3. Xu R, Rai A, Chen M, Suwakulsiri W, Greening DW and Simpson RJ: Extracellular vesicles in cancer-implications for future improvements in cancer care. Nat Rev Clin Oncol 15: 617-638, 2018

4. Cappello F, Logozzi M, Campanella C, Bavisotto CC, Marcilla A, Properzi F and Fais S: Exosome levels in human body fluids: A tumor marker by themselves? Eur J Pharm Sci 96: 93-98, 2017.

5. Melo SA, Luecke LB, Kahlert C, Fernandez AF, Gammon ST, Kaye J, LeBleu VS, Mittendorf EA, Weitz J, Rahbari N, et al: Glypican-1 identifies cancer exosomes and detects early pancreatic cancer. Nature 523: 177-182, 2015

6. Li Y, Zheng Q, Bao C, Li S, Guo W, Zhao J, Chen D, Gu J, He X and Huang S: Circular RNA is enriched and stable in exosomes: A promising biomarker for cancer diagnosis. Cell Res 25: 981-984, 2015
7. Duijvesz D, Luider T, Bangma CH and Jenster G: Exosomes as biomarker treasure chests for prostate cancer. Eur Urol 59: 823-831, 2011.

8. van Niel G, D'Angelo G and Raposo G: Shedding light on the cell biology of extracellular vesicles. Nat Rev Mol Cell Biol 19: 213-228, 2018.

9. Thakur BK, Zhang H, Becker A, Matei I, Huang Y, Costa-Silva B, Zheng Y, Hoshino A, Brazier H, Xiang J, et al: Double-stranded DNA in exosomes: A novel biomarker in cancer detection. Cell Res 24: 766-769, 2014.

10. Kahlert C, Melo SA, Protopopov A, Tang J, Seth S, Koch M, Zhang J, Weitz J, Chin L, Futreal A and Kalluri R: Identification of double-stranded genomic DNA spanning all chromosomes with mutated KRAS and p53 DNA in the serum exosomes of patients with pancreatic cancer. J Biol Chem 289: 3869-3875, 2014.

11. Wan Y, Liu B, Lei H, Zhang B, Wang Y, Huang H, Chen S, Feng Y, Zhu L, Gu Y, et al: Nanoscale extracellular vesicle-derived DNA is superior to circulating cell-free DNA for mutation detection in early-stage non-small-cell lung cancer. Ann Oncol 29: 2379-2383, 2018 .

12. Allenson K, Castillo J, San Lucas FA, Scelo G, Kim DU, Bernard V, Davis G, Kumar T, Katz M, Overman MJ, et al: High prevalence of mutant KRAS in circulating exosome-derived DNA from early-stage pancreatic cancer patients. Ann Oncol 28: 741-747, 2017.

13. Jin Y, Chen K, Wang Z, Wang Y, Liu J, Lin L, Shao Y, Gao L, Yin H, Cui C, et al: DNA in serum extracellular vesicles is stable under different storage conditions. BMC Cancer 16: 753, 2016.

14. Yu M: Somatic mitochondrial DNA mutations in human cancers. Adv Clin Chem 57: 99-138, 2012.

15. Yu M: Generation, function and diagnostic value of mitochondrial DNA copy number alterations in human cancers. Life Sci 89: 65-71, 2011.

16. Reznik E, Miller ML, Şenbabaoğlu Y, Riaz N, Sarungbam J, Tickoo SK, Al-Ahmadie HA, Lee W, Seshan VE, Hakimi AA and Sander C: Mitochondrial DNA copy number variation across human cancers. ELife 5: e10769, 2016.

17. Zhao SY, Yang YF, Liu J, Liu HQ, Ge NJ, Yang HS, Zhang HX and Xing JL: Association of mitochondrial DNA content in peripheral blood leukocyte with hepatitis B virus-related hepatocellular carcinoma in a Chinese Han population. Cancer Sci 102: 1553-1558, 2011.

18. Yin C, Li DY, Guo X, Cao HY, Chen YB, Zhou F, Ge NJ, Liu Y, Guo SS, Zhao Z, et al: NGS-based profiling reveals a critical contributing role of somatic D-loop mtDNA mutations in HBV-related hepatocarcinogenesis. Ann Oncol 30: 953-962, 2019.

19. Mair R, Mouliere F, Smith CG, Chandrananda D, Gale D, Marass F, Tsui DWY, Massie CE, Wright AJ, Watts C, et al: Measurement of plasma cell-free mitochondrial tumor DNA improves detection of glioblastoma in patient-derived orthotopic xenograft models. Cancer Res 79: 220-230, 2019.

20. Guescini M, Genedani S, Stocchi V and Agnati LF: Astrocytes and glioblastoma cells release exosomes carrying mtDNA. J Neural Transm (Vienna) 117: 1-4, 2010.

21. Sansone P, Savini C, Kurelac I, Chang Q, Amato LB, Strillacci A, Stepanova A, Iommarini L, Mastroleo C, Daly L, et al: Packaging and transfer of mitochondrial DNA via exosomes regulate escape from dormancy in hormonal therapy-resistant breast cancer. Proc Natl Acad Sci U S A 114: E9066-E9075, 2017.

22. Satala CB, Jung I, Kobori L, Kovacs Z, Fodor D, Szodorai R and Gurzu S: Benefits of the 8th American joint committee on cancer system for hepatocellular carcinoma staging. J Gastrointest Cancer 2020 (Online ahead of print).

23. Zhu X, Shen H, Yin X, Yang M, Wei H, Chen Q, Feng F, Liu Y, $\mathrm{Xu} \mathrm{W}$ and Li Y: Macrophages derived exosomes deliver miR-223 to epithelial ovarian cancer cells to elicit a chemoresistant phenotype. J Exp Clin Cancer Res 38: 81, 2019.

24. Chen S, Zhou Y, Chen Y and Gu J: Fastp: An ultra-fast all-in-one FASTQ preprocessor. Bioinformatics 34: i884-i890, 2018.

25. Li H, Handsaker B, Wysoker A, Fennell T, Ruan J, Homer N, Marth G, Abecasis G and Durbin R; 1000 Genome Project Data Processing Subgroup: The sequence alignment/map format and SAMtools. Bioinformatics 25: 2078-2079, 2009.

26. McKenna A, Hanna M, Banks E, Sivachenko A, Cibulskis K, Kernytsky A, Garimella K, Altshuler D, Gabriel S, Daly M and DePristo MA: The genome analysis toolkit: A MapReduce framework for analyzing next-generation DNA sequencing data. Genome Res 20: 1297-1303, 2010.

27. Maas SLN, Breakefield XO and Weaver AM: Extracellular vesicles: Unique intercellular delivery vehicles. Trends Cell Biol 27: 172-188, 2017. 
28. Jahr S, Hentze H, Englisch S, Hardt D, Fackelmayer FO, Hesch RD and Knippers R: DNA fragments in the blood plasma of cancer patients: Quantitations and evidence for their origin from apoptotic and necrotic cells. Cancer Res 61: 1659-1665, 2001.

29. Mouliere F, Chandrananda D, Piskorz AM, Moore EK, Morris J, Ahlborn LB, Mair R, Goranova T, Marass F, Heider K, et al: Enhanced detection of circulating tumor DNA by fragment size analysis. Sci Transl Med 10: eaat4921, 2018.

30. Underhill HR, Kitzman JO, Hellwig S, Welker NC, Daza R, Baker DN, Gligorich KM, Rostomily RC, Bronner MP and Shendure J: Fragment length of circulating tumor DNA. PLoS Genet 12: e1006162, 2016.

31. Patrushev M, Kasymov V, Patrusheva V, Ushakova T, Gogvadze V and Gaziev A: Mitochondrial permeability transition triggers the release of mtDNA fragments. Cell Mol Life Sci 61: 3100-3103, 2004

32. Qu C, Zhang S, Li Y, Wang Y, Peppelenbosch MP and Pan Q: Mitochondria in the biology, pathogenesis, and treatment of hepatitis virus infections. Rev Med Virol 29: e2075, 2019.

33. McArthur K, Whitehead LW, Heddleston JM, Li L, Padman BS Oorschot V, Geoghegan ND, Chappaz S, Davidson S, Chin HS, et al: BAK/BAX macropores facilitate mitochondrial herniation and mtDNA efflux during apoptosis. Science 359: eaao6047, 2018.

34. Zhang X, Wu X, Hu Q, Wu J, Wang G, Hong Z and Ren J; Lab for Trauma and Surgical Infections: Mitochondrial DNA in liver inflammation and oxidative stress. Life Sci 236: 116464, 2019.
35. Hsu CC, Lee HC and Wei YH: Mitochondrial DNA alterations and mitochondrial dysfunction in the progression of hepatocellular carcinoma. World J Gastroenterol 19: 8880-8886, 2013.

36. Lee HC, Li SH, Lin JC, Wu CC, Yeh DC and Wei YH: Somatic mutations in the D-loop and decrease in the copy number of mitochondrial DNA in human hepatocellular carcinoma. Mutat Res 547: 71-78, 2004

37. Baysa A, Fedorov A, Kondratov K, Ruusalepp A, Minasian S, Galagudza M, Popov M, Kurapeev D, Yakovlev A, Valen G, et al: Release of mitochondrial and nuclear DNA during on-pump heart surgery: Kinetics and relation to extracellular vesicles. J Cardiovasc Transl Res 12: 184-192, 2019.

38. Bernard V, Kim DU, San Lucas FA, Castillo J, Allenson K, Mulu FC, Stephens BM, Huang J, Semaan A, Guerrero PA, et al: Circulating Nucleic Acids Are Associated With Outcomes of Patients With Pancreatic Cancer. Gastroenterology 156: 108-118, 2019.

39. Weerts MJA, Timmermans EC, van de Stolpe A, Vossen RHAM, Anvar SY, Foekens JA, Sleijfer S and Martens JWM: Tumor-specific mitochondrial DNA variants are rarely detected in cell-free DNA. Neoplasia 20: 687-696, 2018.

(i) (9) This work is licensed under a Creative Commons Attribution-NonCommercial-NoDerivatives 4.0 International (CC BY-NC-ND 4.0) License. 